\title{
Acoustic microcavitation: its active and passive acoustic detection
}

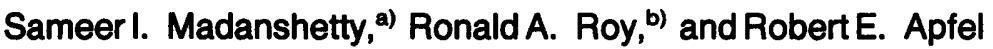 \\ Department of Mechanical Engineering, Center for Ultrasonics and Sonics, Yale University, \\ 9 Hillhouse Avenue, New Haven, Connecticut 06520
}

(Received 1 February 1990; revised 23 May 1991; accepted 24 May 1991)

\begin{abstract}
In this work acoustic microcavitation in water is studied primarily at $0.75 \mathrm{MHz}$ and $1 \%$ duty cycle. To detect cavitation, two kinds of acoustic detectors are used. The first one is an unfocused, untuned 1-MHz receiver transducer that serves as a passive detector. The other one is a focused 30-MHz transducer that is used in pulse-echo mode and is called the active detector. Cavitation itself is brought about by a focused PZT-8 crystal driven in pulse mode. The active detector is arranged confocally with respect to the cavitation transducer. Both the interrogating pulse and the cavitation pulse arrive simultaneously at the common focus, which is the region of cavitation. With the test chamber filled with clean water, no cavitation is observed, even when the cavitation transducer is driven to give its peak output of 22 bar peak negative. Cavitation is, however, observed when polystyrene microparticles are added to the host water. Our view of how these smooth, spherical, monodispersed microparticles give rise to cavitation is described with some estimates. An attempt has been made to understand whether the presence of "streaming" affects the thresholds, and it has been found that the active detector field affects the cavitation process.
\end{abstract}

PACS numbers: 43.35.Yb, 43.35.Ei

\section{INTRODUCTION}

Recently, an acoustic backscattering technique for the detection of transient cavitation produced by microsecond pulses of ultrasound was discussed by Roy et al. ${ }^{1}$ Further investigation on the active detection of transient cavitation is reported here.

Figure 1 sets the context. When the test chamber, containing microparticles suspended in a clean water host, is irradiated with short acoustic pulses from the $0.75-\mathrm{MHz}$ focused cavitation transducer, the cavitation thresholds indicated by the unfocused passive transducer are around $15 \mathrm{bar}$ peak negative. If the $30-\mathrm{MHz}$ focused active transducer is switched on to operate in the pulse-echo mode, the measured thresholds are about 7 bar peak negative. This observation in itself, may amply speak for the superior sensitivity of the active detector. However, if the thresholds are measured with the passive transducer, while the active transducer is left switched on, the passive thresholds drop to around 8 bar peak negative. This difference in the measured passive thresholds, 15 bar peak negative with the active detector off, and 8 bar peak negative with the active detector on, suggests that the cavitation environment is being influenced by the presence of the active detector detection field. In the present experimental setup, the active detector focal pressure amplitudes are invariably less than 0.5 bar peak negative. This in itself, even with the crudest superposition, cannot account for the lowering of thresholds from 15 bar peak negative to 8 bar peak negative. In fact, it becomes increasingly difficult to

\footnotetext{
a) Current address: Mechanical and Aerospace Engineering, Boston University, 110 Cummington Street, Boston, MA 02215.

b) Current address: National Center for Physical Acoustics, P. O. Box 847, University, MS 38677.
}

initiate cavitation at increased acoustic frequencies, and it is found that the active detector does not cause cavitation on its own. It is thought that the streaming caused by this highfrequency focused device may have some part to play. This lead comes from a previous observation.

The apparatus used by Atchley $e t$ al. ${ }^{2}$ consisted of a chamber filled with a host fluid: distilled water. In this chamber a jet of the same host fluid was arranged to run through the focus of the cavitation transducer. Cavitation was brought about by running polystyrene microparticles through this jet. While measuring the background cavitation activity, it had been observed that the cavitation thresholds

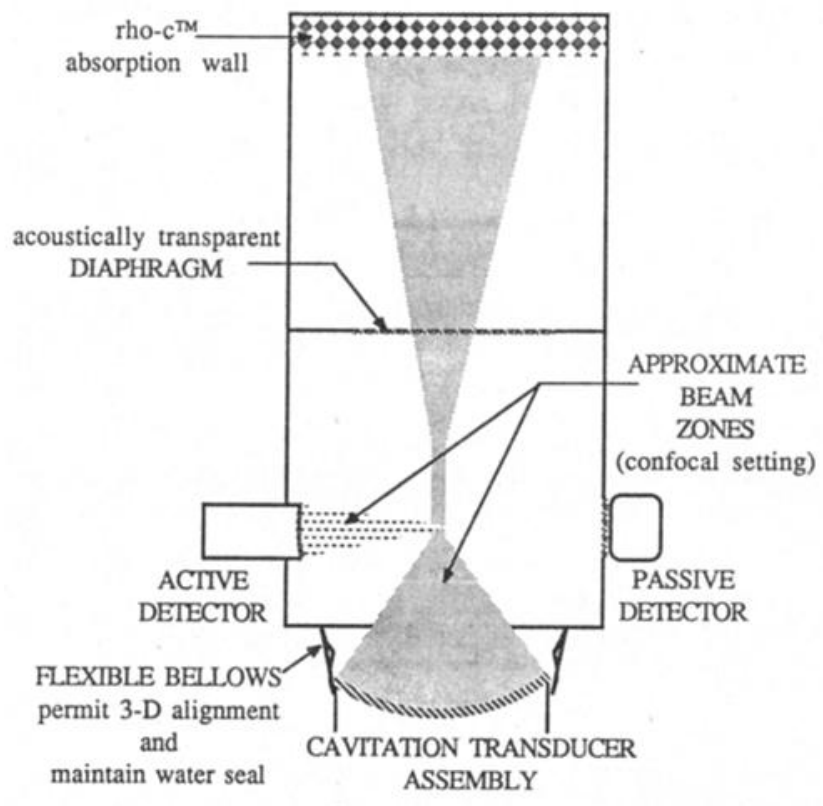

FIG. 1. Schematic of the test cell. 
for a quiescent body of water were higher than those in the presence of the jet, running no particles. This was suspected then to be due to dirt being insidiously convected into the focal region by the jet. ${ }^{3}$ Further, in these experiments the role of the latex microspheres used in the core flow of the jet in promoting cavitation was not fully understood.

Therefore, a new system was built to maintain and monitor extreme cleanliness. Without intentional seeding, no cavitation could be detected even at pressure amplitudes in excess of 22 bar peak negative, thus permitting controlled experiments to study the efficacy of nucleation by foreign particles.

An understanding of the role of the active detector in facilitating cavitation is one of the objectives of this work. A possible scenario is proposed to explain how smooth spherical monodispersed microparticles can give rise to cavitation. Threshold as a function of the particle and pulse parameters are also reported.

\section{EXPERIMENTAL SETUP}

The apparatus consists of the following subsystems: (1) clean water system, (2) test cell, (3) cavitation signal, (4) detectors, and (5) data acquisition.

\section{A. Clean water system}

The main reservoir is made of Plexiglass and has a capacity of $12 \ell$, which is about seven times the capacity of the test cell. Once the reservoir is filled with water, it stays isolated throughout the processing; it communicates with the ex-

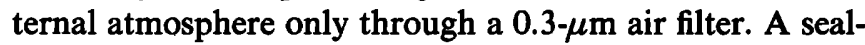
less magnetically driven variable flow centrifugal pump, a deionizing column, an organic removal column, and a 0.2 $\mu \mathrm{m}$ industrial grade Fin-L-Filter ${ }^{\mathrm{TM}}$ are connected in series with the reservoir to form a closed loop. The pump is a lowhead, large flow type and the flow resistance offered by the filter is only marginal. The "once through" circulation time is around $2 \mathrm{~min}$. The simplicity and the flexibility of the clean water system is apparent from the schematic Fig. 2. Use of polypropylene quick-connect valves minimizes the tubing length, number of direction control valves, and section transitions (possible sites for dirt stagnation). They reduce the pumping pressure drops to a minimum, provide clean ducting, and are leakproof even under vacuum. Clean distilled water $(17.8 \mathrm{M} \Omega / \mathrm{cm})$ from the Barnstead D2791 NANOpure ${ }^{T M}$ unit is introduced into the reservoir through the Fin-L-Filter ${ }^{\mathrm{TM}}$ filter. Typically a filtration cycle of $1 \mathrm{~h}$ is adequate to ensure a reservoir full of clean water. The filtration integrity of the system is better than an industrial grade one. In fact, the water is so clean that no cavitation is detected even when the cavitation transducer is driven to yield pressures in excess of 22 bar peak negative, even with the active detector set to its highest sensitivity.

\section{B. Test cell}

The test cell is divided into two compartments so that the rho- $\mathrm{c}^{\mathrm{TM}}$ impedance matching rubber wall immersed in water provides a nonreflecting boundary while remaining isolated from the cavitation chamber. An acoustically trans-

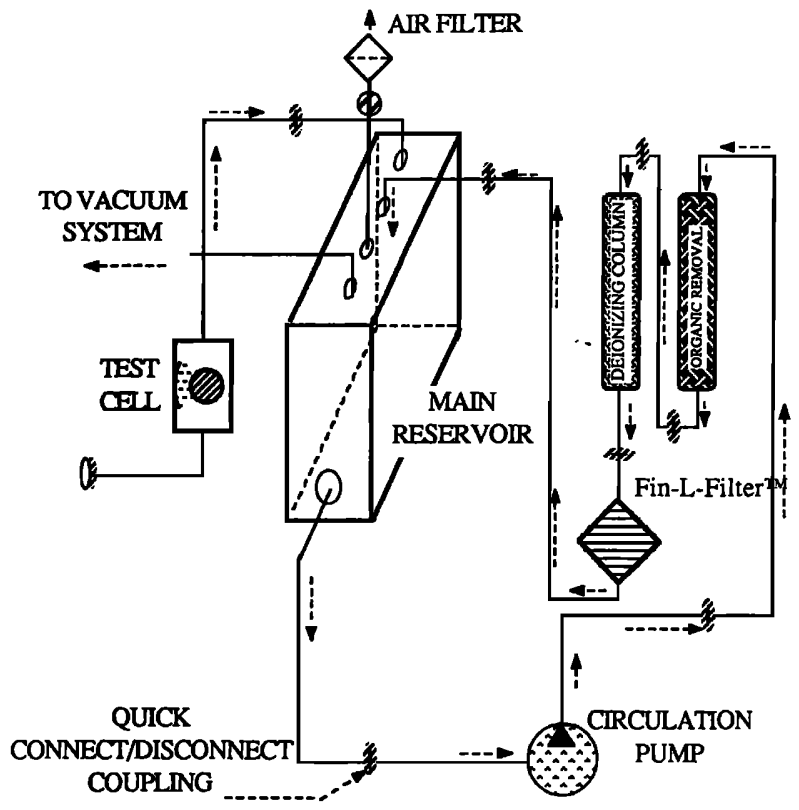

FIG. 2. Schematic of the clean water system. Particulate contaminants are removed from water by repeated filtration through a closed loop. Clean water provides a cavitation-free host environment for the experiments. The quick connect/disconnect couplings permit easy selection of the various components as needed.

parent 50-mm-diam, $12-\mu \mathrm{m}$-thick stainless shim stock forms a watertight seal between the two compartments. A temperature probe inserted in the rho- $\mathrm{c}^{\mathrm{TM}}$ compartment monitors the test temperature. The main cavitation cell is flexibly connected to the cavitation transducer mounted on the translation stages to permit geometric alignment. The main cavitation transducer is a PZT-8, 37-mm-diam spherical segment of 50-mm focal length. It is air backed and operated at its resonance frequency of $0.75 \mathrm{MHz}$. On the side wall of the cavitation cell, the active detector $-30-\mathrm{MHz}$ lithium niobate crystal bonded to a $25-\mathrm{mm}$ focal length styrene lens - is precisely located in a sealing sleeve so that its focus is on the cell center line, and its beam axis is square to the axis of the main cavitation transducer. On the opposite wall directly across from the active detector is a 1.6-mm-thick Plexiglas window, where the passive detector $(1-\mathrm{MHz}, 19-\mathrm{mm}$, broadband, nonfocused transducer) can be positioned from the outside in approximate alignment with the active detector. Ultrasonic gel is used to couple the passive detector to the test cell. The cell can be effortlessly connected into the clean water system through the quick connects provided. During the test runs the cavitation cell top is connected to the reservoir top so that they share a common exposure to the atmosphere through the submicronic air filter.

\section{Cavitation signal generation}

The cavitation signal is a tone burst-a continuous sine wave, amplitude modulated by a non-negative going square wave. Figure 3 illustrates how the cavitation signal is generated. The General-Radio oscillator is tuned to $755.2 \mathrm{kHz}$, the maximum power frequency of the main cavitation transducer. A cavitation module generates the needed square waves to control the duty cycle and also provides the exter- 


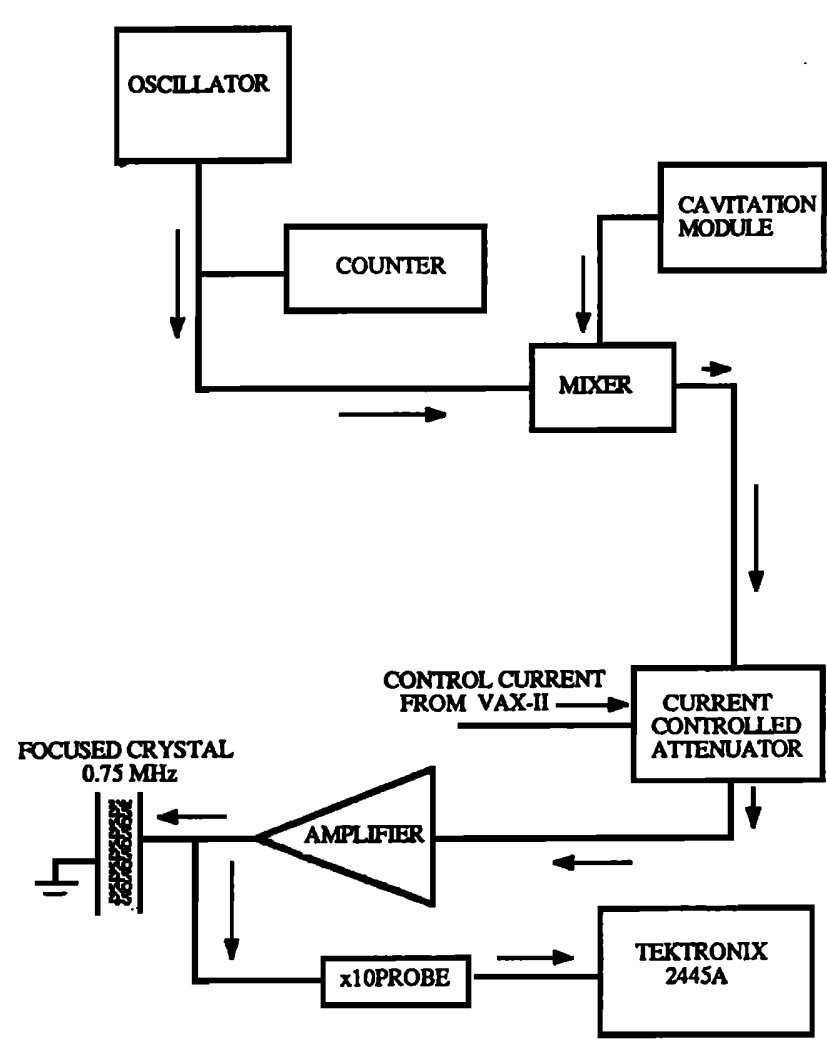

FIG. 3. Schematic of cavitation signal generation.

nal trigger signals for the scope. The amplitude modulated output from mixers is fed to a current controlled voltage attenuator. The controlling current is generated by a simple voltage-to-current converter driven by the voltage output from the VAX-II DAC. The output is amplified by a ENI240L power amplifier before it drives the main cavitation transducer. Impedance matching in this case was not very critical. Due to the low duty cycle, the amplifier was never driven to its full capacity even near saturation voltages (in the present case $330 \mathrm{~V}$ peak to peak).

\section{Detectors}

The active detector is a focused $30-\mathrm{MHz}$ transducer that transmits a pulse in the transmit mode and picks up the return echo signal in the receive mode, should it encounter any suitable target. The mode selection is controlled by a transmit-receive switch (Ref. 1). The active detector pulse can be precisely synchronized in time and space with respect to the main cavitation pulse, so that both the pulses arrive simultaneously at the common focus. The spatial confocal setting of the transducers calls for centralizing the $0.2-\mathrm{mm}$ focal spot of the active detector within the 2-mm-diam focal spot of the main cavitation transducer. The small focal spot of the active detector enables us to probe the focal field of the cavitation transducer. The detection frequency of $30-\mathrm{MHz}$ is expected to yield more information compared to a lower detection frequency, should the interrogating pulse encounter a cavitation event. In fact, a $30-\mathrm{MHz}, 10-\mu \mathrm{s}-$ long detector pulse has about 20 full cycles to strobe the growth of a single cycle cavitation event caused by the $0.75-\mathrm{MHz}$ cavitation frequency.
The passive detector, which does not actively interrogate the cavitation zone, is an unfocused, untuned 1-MHz transducer. It is coupled to the test cell with ultrasonic gel. It is located directly across from the active detector.

\section{E. Data acquisition}

A typical cavitation run involves the following sequence of actions on the part of the experimenter.

(i) Ramp up the input voltage to the cavitation transducer at a predetermined rate, and observe the scope trace of the selected detector for a cavitation event.

(ii) On sighting the cavitation event, stop the ramping and hold the voltage level steady (see Sec. II A).

(iii) Read the voltage and hence the cavitation pressure amplitude-the threshold (see Appendix A for transducer calibration).

(iv) Drop the voltage to zero, and wait for some predetermined time before commencing step (i) ramping for the next reading.

These steps have been incorporated into a fortran routine, and the entire procedure of ramping, freezing, and reading voltages can be controlled by a VAX-II via the Data Translation DT2768 board.

\section{EXPERIMENTAL PROCEDURES}

Cavitation results delicately depend upon the experimental conditions. Cavitation is particularly affected by the cleanliness of the experimental environment; every factor must be monitored and carefully accounted for. Ingress of dirt, unattended atmospheric leaks, unknown dissolved constituents, foreign organic presence, and unmonitored ionic imbalances can insidiously affect cavitation activity and make the experiments unrepeatable. After the system was set up, it took us more than a month of trials to identify, examine, and standardize the test procedures (Ref. 3 ).

System start-up procedures include ensuring the cleanliness integrity of the host water, setting of the detectors and alignments, calibration of the main cavitation transducer, estimation of the streaming velocities, particle preparation, establishing the test parameters, and proofing the data acquisition system. Transducer calibration and the estimation of the streaming velocities are described briefly in the appendices.

In all the experiments described in this paper, latex particles manufactured by the Interfacial Dynamics Corporation have been used. These are carboxyl polystyrene latex spheres suspended in distilled water and are surfactant free. We used three sizes of particles: $0.245,0.481$, and $0.984 \mu \mathrm{m}$ in diameter. These are monodispersed, spherical, and smooth. Extensive observation under the scanning electron microscope did not reveal any surface flaws or crevices down to about $500 \AA$, the resolution limit of the device. The specific area based on the adsorption studies also was close to the geometric surface area calculated from the sphere assumption. The density of these polystyrene particles at $20^{\circ} \mathrm{C}$ is $1.05 \mathrm{~g} / \mathrm{cc}$. From the manufacturer's information about the solid fraction of the colloidal suspension, we could easily control the particle number density used in the various experiments. 
Typically at end of the day's trials the system would be flushed clean, and the reservoir topped with freshly distilled water and filtered through for at least $1 \mathrm{~h}$, and then left unperturbed for the night after the air filter was closed off to secure complete isolation. On the following morning, while the electronics warmed up (oscillators were never switched off), the dissolved air content of the reservoir water was measured using the Orion Research oxygen probe. (The dissolved air concentrations mentioned in this paper are within $\pm 2 \%$ of the stated values and remain fairly constant during the day long trials). The test cell was then gently filled with the clean water from the reservoir and checked for background cavitation activity with both the active and the passive detectors. The main cavitation transducer would be set to $10-\mu$ s pulse width, $1-\mathrm{kHz}$ PRF, and the active detector would be set at 10- $\mu$ s pulse width, 1-kHz PRF and to its highest detection voltage setting possible in the present apparatus, and tracking the main cavitation transducer. The detector pulse is suitable delayed to ensure that both the detector and the cavitation pulse arrive simultaneously at the common focus. Based on trials, the test parameters are fixed at ${ }^{3}$ linear ramp rate of $100 \mathrm{~V}_{\mathrm{pp}}$ per min (i.e., 7 bar peak negative per min or $1.2 \mu$ bar peak negative per cavitation pulse), from 50 to $300 \mathrm{~V}_{\mathrm{pp}}$ (or the cavitation event) input to the main cavitation transducer, and a dwell of $10 \mathrm{~s}$ between two readings. Thirty readings were taken per set. Nine to fourteen sets could be completed in a day without tiring the eyes; each event has to be sighted visually on the scope screen. As for the background cavitation activity, we would not proceed with the day's test unless we got a clean record: no cavitation events in 20 counts with both the active and the passive detectors.

It has been verified that the sequence of varying the streaming velocities does not affect the thresholds. For a given setting of the streaming velocity, the thresholds at the beginning of the day differ little from those at the end of the day. This variation in thresholds is less than the day-to-day variation in repeat runs, and the worst variation in thresholds measured in runs separated by 2 weeks is within a couple of atmospheres.

Before giving the experimental results, we briefly describe the nature of the cavitation events as seen on the Tektronix 2445A, dual time base 150-MHz analog oscilloscope.

\section{A. Sighting of cavitation events}

In this work, every threshold measurement was made only after a crucial decision-have I sighted a cavitation event?-had been taken by the experimenter. Since more than 30000 such decisions were made during the course of this investigation, in this section we make some observations regarding the recognition of the cavitation event. Typical scope traces of the events can be found in Ref. 1.

\section{B. Passive detector events}

The trace of the passive detector, when displayed on the A-time base, is an outline of hills and valleys, almost symmetric about the ground trace. The region within the outline is bright and renders any signal less than the outline unre- cognizable. On the scope this background signal is a region of even, steady brightness. When the voltage input to the cavitation transducer is ramped linearly, the steady reverberant background displayed on the scope increases in proportion. So, in order to be seen, a cavitation event, when it occurs, should tower above the reverberant background. This is often the case because bubbles are strong scatterers and, unless very weak, the event does manifest itself clearly.

Since almost all the cavitation occurs in the focal region of the main cavitation transducer, and that region is spatially fixed relative to the passive transducer, the site of appearance of the events on the scope is also fixed. On the A-time base, along with the main event, one also sees the various secondary echoes down the time axis. Therefore, to isolate the main event and enhance the clarity, we invoke the B-time base, and locate the magnifying window around the region of occurrence of the main event.

Eye training in the case of the passive detector signals is aided by the fact that the background that is to be visually subtracted is constant. Further, the cavitation event occurs at a fixed location and is distinct from any stray glitches (switching transients from the neighboring experiment) that sometimes appear. The towering differential of the event above the background depends on the strength of the event and also the threshold value. Events in degassed water (with any particles) appear to be strong, crisp, and sharp. The reverberant background is little affected by the kind of nuclei used in the tests. The enveloping outline is almost similar in all experiments.

\section{Active detector events}

To be able to detect the cavitation events as registered by the active detector, one has to invoke the B-time base. The magnifying window is located to span the detector receive gate. In this window there is usually a considerable amount of activity. First, there is a basic background rippling noise, which is dependent on the nature, size, and the number density of the particles in the cell. Superimposed on this background noise is what we call "the elephant activity" (cf. Appendix B). "Elephants" in contrast to the crisp transient cavitation events are wide backscattered echoes that slowly move down the scope trace. Small elephants (about twice the noise level) continuously move down stream, often changing their size slightly as they move out. Elephants are as wide as the active detector pulse. The only chance of missing an event is when a large elephant scans through the screen and momentarily blocks the cavitation site from observation. Since this is not a frequent occurrence, it does not cause serious errors.

The cavitation event itself occurs at a fixed location, for a given pulse and delay setting. It is very short lived, and often looks like a couple of cycles, with a characteristic signature, apparently superimposed with the cavitation transducer frequency. The event is quite transient, sharp, and unmistakable in spite of the busy traffic along the time axis. As to the character of the event, previously made comments in connection with the passive events apply.

Active detection strains the eyes more than passive detection and may affect accuracy in longer test runs. 


\section{EXPERIMENTS AND RESULTS}

\section{A. Active and passive thresholds}

Figure 4 is a typical comparison of the active and the passive detectors. This test was conducted with $0.245-\mu \mathrm{m}$ polystyrene spheres, at a number density of $2.5 \times 10^{8}$ particles/cc. The dissolved air concentration of the test cell water was $84 \%$ of saturation. Information from this plot was used in the introduction to bring out the central question of this investigation: Why are the observed thresholds lower in the presence of the active detector field?

The active detector is a high-frequency device and, when at the highest setting (possible with our apparatus) of $46 V_{p p}$ and $10-\mu s$ pulse width, generates a pressure amplitude of 0.5 -atm peak negative at its focus. We have checked carefully to see if the active detector can cause cavitation on its own when the main cavitation field is off and have found no evidence of such cavitation.

Being a high-frequency focused device, the active detector, however, causes acoustic streaming. We are using streaming velocity as a convenient abscissa to represent the different settings of the active detector. The streaming velocity depends on the setting of the active detector. We commonly use the following four pulse-width/voltage settings: $2 \mu \mathrm{s} / 25 \mathrm{~V}_{\mathrm{pp}}, \quad 5 \mu \mathrm{s} / 25 \mathrm{~V}_{\mathrm{pp}}, \quad 4 \mu \mathrm{s} / 46 \mathrm{~V}_{\mathrm{pp}}, \quad 10 \mu \mathrm{s} / 46 \mathrm{~V}_{\mathrm{pp}}$, which correspond to mean streaming velocities of $0.27,0.48$, 1.86 , and $3.25 \mathrm{~cm} / \mathrm{s}$, respectively. Note that because of the ease of measurement, these detector voltages were measured at the amplifier output (Ref. 3 ). True detector voltages are obtained after accounting for the 6-dB transmit signal insertion loss. So the actual detector voltages are half the values stated here.

The plot shows that both the detectors track the trend in the threshold variation with respect to the streaming velocity. At higher streaming velocities the two trends are almost parallel, indicating that the two detectors elicit the essential threshold information. The passive detector can also measure the thresholds at zero streaming velocity-active detector switched off.

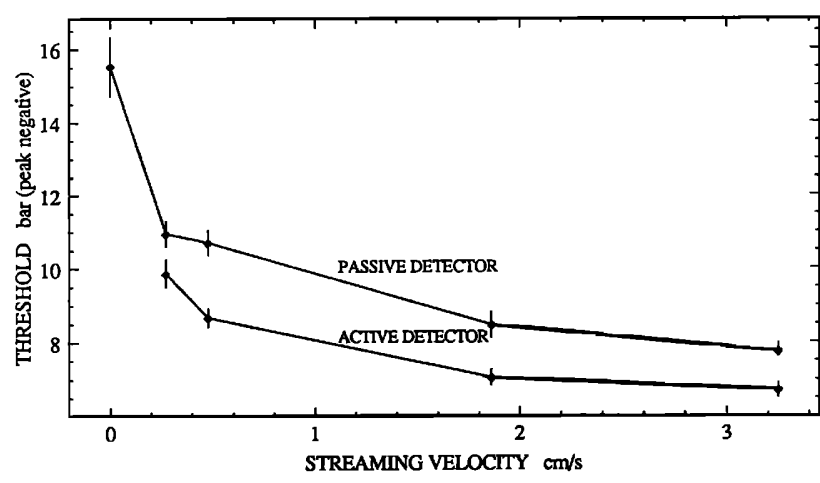

FIG. 4. A comparison of the two detectors. Observed passive thresholds are higher than active thresholds. The plots also indicate the threshold trend as the streaming velocity is increased. Cavitation transducer: $10-\mu$ s pulse, $1-$ kHz PRF, linear ramp, and confocal setting. Test cell: $0.245-\mu \mathrm{m}$ polystyrene spheres, $2.5 \times 10^{8}$ particles/cc, water ( $84 \%$ dissolved air saturation).

\section{B. Effect of particle concentration}

Both the active and the passive thresholds were measured as a function of the streaming velocity, for increasing particle concentration. The aim was to seek a possible saturation concentration. At such concentration, it might be expected that the streaming effect (flowing in of more nuclei) of the active detector would be of little significance since there would always be enough nuclei in the focal region to cavitate from.

In the experiment the particle size was kept constant $(0.245-\mu \mathrm{m}$ polystyrene), while the concentration was increased from $1.2 \times 10^{8}$ particles/cc to $7.8 \times 10^{9}$ particles/cc, a 64-fold increase in number density. The dissolved air saturation varied between $70 \%$ to $85 \%$ over the test runs. Figures 5 and 6 indicate how the thresholds change with increase in concentration. The curves are plotted with respect to the normalized number density as the parameter, the normalization is with respect to the smallest particle concentration. The particle concentration could not be increased any further because the background backscatter became excessive, making active detector threshold identification difficult.

It is evident from the plots (Figs. 5 and 6) that the saturation concentration is not apparently reached; the passive thresholds for zero streaming continue to drop with increase in concentration. However, the plots show that the dependence of the thresholds on streaming velocity decreases as the particle concentration increases. Even at high particle concentrations, the passive thresholds in the presence of the active detector field are lower.

At the lowest particle concentration, the interparticle separation is about $20 \mu \mathrm{m}$, or 80 particle diameters. At the highest particle concentration, the interparticle separation decreases to about 20 diameters. The particles do not agglomerate even in the concentrations as supplied by the manufacturer, which are about 1000-fold higher than the highest concentrations used in the above tests.

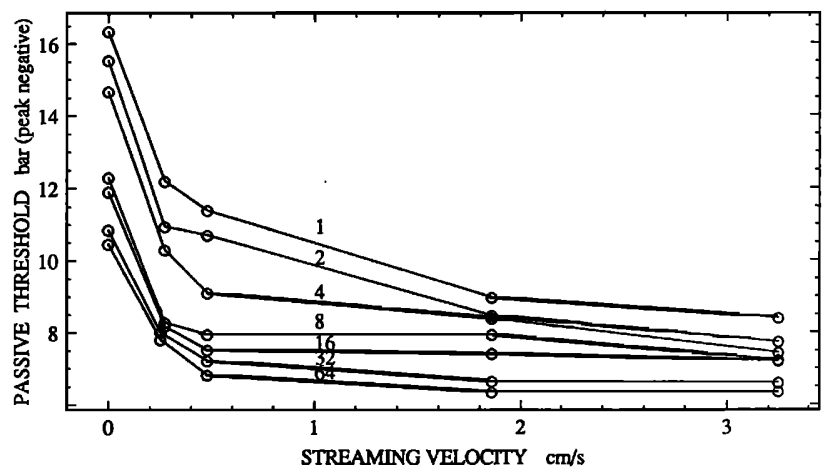

FIG. 5. Passive threshold variation. For a fixed particle concentration, as the streaming velocity is increased, lower passive thresholds are observed. The particle concentration is varied from $1.2 \times 10^{8}$ particles/cc to $7.8 \times 10^{9}$ particles/cc-a variation of $1: 64$. The lowest particle concentration corresponds to an interparticle separation of about $20 \mu \mathrm{m}$ or equivalently 80 particle diameters. At the highest concentration, the particles are separated by about 20 particle diameters. Cavitation transducer: $10-\mu$ s pulse, $1-\mathrm{kHz}$ PRF, linear ramp, and confocal setting. Test cell: $0.245-\mu \mathrm{m}$ polystyrene spheres, water ( $70 \%$ to $85 \%$ dissolved air saturation). 


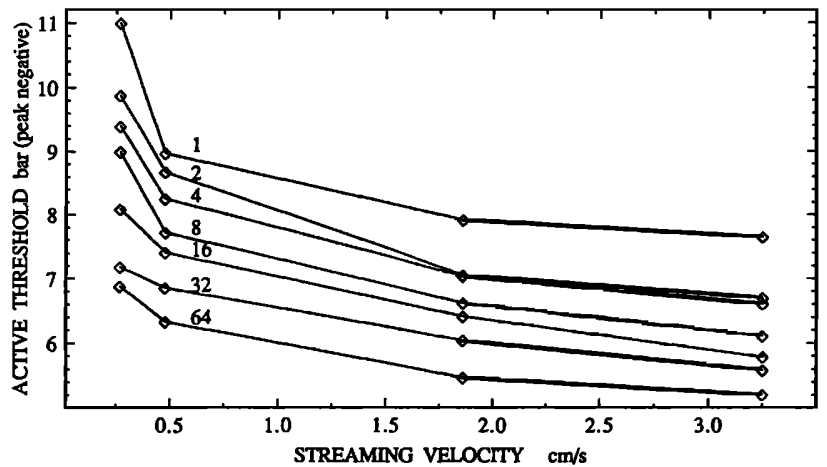

FIG. 6. Active threshold variation. Same test conditions as Fig. 5.

\section{Thresholds for constant particle flux}

Since the effect of streaming is to inject particles in the cavitation field, it would be worthwhile to keep the particle flux constant and observe whether the cavitation thresholds remain constant. In this test the product of the streaming velocity and the particle concentration was kept constant. The velocity was varied by varying the pulse width of the active detector, while keeping its voltage constant at $46 \mathrm{~V}_{\mathrm{pp}}$. The particle size used was $0.245 \mu \mathrm{m}$, and the particle density at the normal concentration was $1.5 \times 10^{9}$ particles $/ \mathrm{cc}$. The velocity at the normal concentration was $0.81 \mathrm{~cm} / \mathrm{s}$. The dissolved air saturation in the test cell was $74 \%$.

Figure 7 shows the measured active thresholds along with the standard error bars. The first two points show a remarkable constancy of thresholds for constant particle flux, which indicates that streaming does convect nuclei into the cavitation zone. The third point indicates that the thresholds have dropped, apparently indicating that the process of nucleation has become more effective. However, the null hypothesis could not be rejected at $99 \%$ level of confidence. From the above one can expect the thresholds to remain the

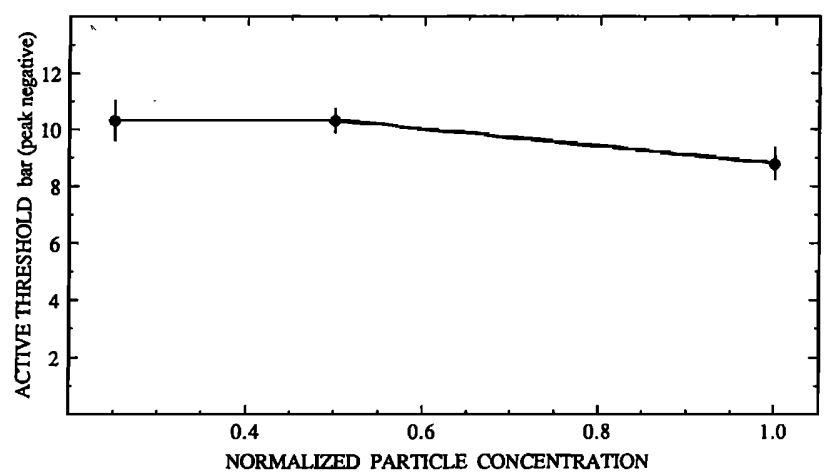

FIG. 7. Active thresholds for constant particle flux. A constant streaming velocity-particle concentration product suggests that the number of potential cavitation nuclei flowing into the cavitation focal zone is constant, and therefore the observed threshold should remain the same. The plot shows that the threshold variation is about 1.5 bar. Particle concentration has been normalized with respect to $1.5 \times 10^{9}$ particles/cc. Streaming velocity at normal concentration is $0.81 \mathrm{~cm} / \mathrm{s}$. Active detector setting: $2.5,5$, and $10-\mu \mathrm{s}$ pulse widths at $1,0.5$, and 0.25 normal concentrations, respectively, $46 V_{p p}$, confocal setting. Cavitation transducer: $10-\mu$ s pulse, $1-\mathrm{kHz}$ PRF. Test cell: $0.245-\mu \mathrm{m}$ polystyrene spheres, water ( $74 \%$ dissolved air saturation). same for constant particle flux, particularly when the particle concentrations are lower. Apart from keeping the particle flux constant, the detector voltage was also held fixed and the streaming velocity changed by changing the pulse width. This suggests the following experiment.

\section{Thresholds for constant streaming velocity}

Since the observed streaming velocity is proportional to the product of the pulse width and the square of the detector voltage, by suitably adjusting the factors, we have found that one can achieve the same streaming velocity for different settings of the active detector. In these tests the particle concentration was kept constant along with the streaming velocity $(1.86 \mathrm{~cm} / \mathrm{s})$, and the thresholds were measured for different settings of the active detector. The particles used were $0.245-\mu \mathrm{m}$ polystyrene, and the dissolved air concentration was around $82 \%$. Figure 8 depicts the effects at a particle concentration of $7.9 \times 10^{9}$ particles/cc. The plot indicates that the observed thresholds are essentially the same at the various settings of the active detector. This lends support to the observation of constant thresholds at constant particle flux.

\section{E. Effect of the screen on threshold}

Thus far the experiments conducted attempt to study the effect of the streaming velocity and the particle concentration on the observed thresholds. Even though constant particle flux tends to give reasonably constant thresholds, we are yet to answer the central question: Why are the observed passive thresholds lower in the presence of the active detector field?

We have not yet studied the effect of the active detector field isolated from its streaming influence. We have, however, through extensive trials seen no evidence of cavitation due to the active detector acting alone. The active detector is unlikely to cause cavitation on its own because of the following: (1) At higher frequencies (greater than $3 \mathrm{MHz}$ ), as noted by Neppiras, ${ }^{4}$ it becomes exceedingly difficult to bring

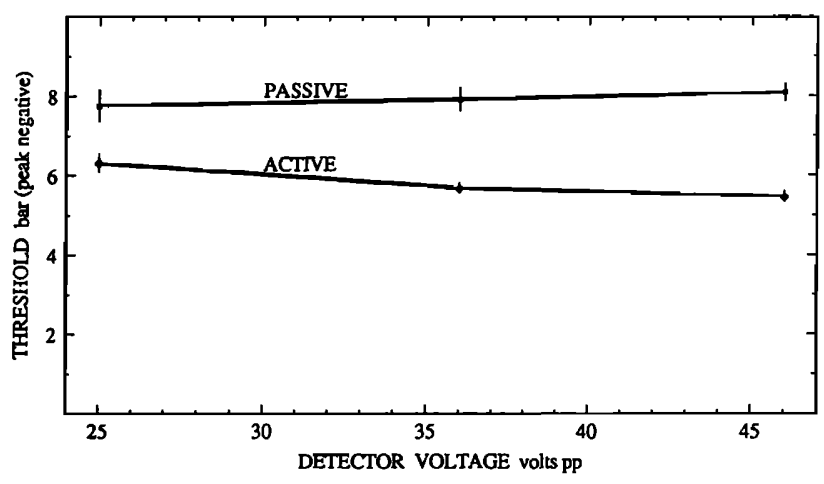

FIG. 8. Thresholds for constant mean streaming. It is observed that the streaming velocity can be kept constant by keeping the (detector pulse width-voltage ${ }^{2}$ ) product constant. Here, the mean streaming velocity was maintained at $1.86 \mathrm{~cm} / \mathrm{s}$. The observed threshold variation is within an atmosphere for both the detectors. (The particle flux is also constant.) Cavitation transducer: $10-\mu$ s pulse, $1-\mathrm{kHz}$ PRF, linear ramp, and confocal setting. Test cell: $0.245-j i \mathrm{~min}$ polystyrene spheres, $7.9 \times 10^{9}$ particles $/ \mathrm{cc}$, water (82\% dissolved air saturation). 
about cavitation, primarily because the bubble does not have enough time to grow. The active detector frequency in the present setup is $30 \mathrm{MHz}$. (2) The pressure amplitudes at which the active detector operates are considerably smaller than the measured thresholds.

We have yet to establish conclusively that the active detector acoustic field is innocuous and that only its streaming effect is responsible for lowering of thresholds. We therefore conducted the present experiment. We put a piece of Saran $\mathrm{Wrap}^{\mathrm{TM}}$ (about $8 \mu \mathrm{m}$ thick) as far in front of the active detector as possible, without infringing into the cone of convergence of the cavitation transducer. This impervious screen discourages the buildup of the streaming field due to the active detector, while remaining nearly transparent to the acoustic field due to the active detector. In this configuration, since the streaming is greatly discouraged, the thresholds should not decrease with the increased active detector pulse/voltage setting if streaming is the sole cause for lowering the observed thresholds. We measure the passive thresholds both with and without the screen, while keeping the test cell environment the same.

Figure 9 clearly shows that the acoustic field of the active detector does play a role in the cavitation process; it helps lower the thresholds. Both the curves converge, as they should, at the 0 setting corresponding to the active detector in the off state.

In order to understand the mechanics of participation of the active detector; we need to make a few estimates. We refer to Figs. 5 and 6 for information regarding the number densities of particles. The focal zone of the main cavitation transducer can be approximated as a cylinder of diameter 2 $\mathrm{mm}$ and length $9 \mathrm{~mm}$. At any time in this volume of $28 \mathrm{~mm}^{3}$ there will be approximately $3.5 \times 10^{6}$ particles even at the lowest concentration. Similar estimates indicate that at the highest streaming velocity if $3.25 \mathrm{~cm} / \mathrm{s}$ the active detector can convect an estimated $1.8 \times 10^{5}$ particles per second into the focal zone. Since the cavitation pulse is $10 \mu$ s long, as is the active detector at the setting corresponding to $3.25 \mathrm{~cm} / \mathrm{s}$,

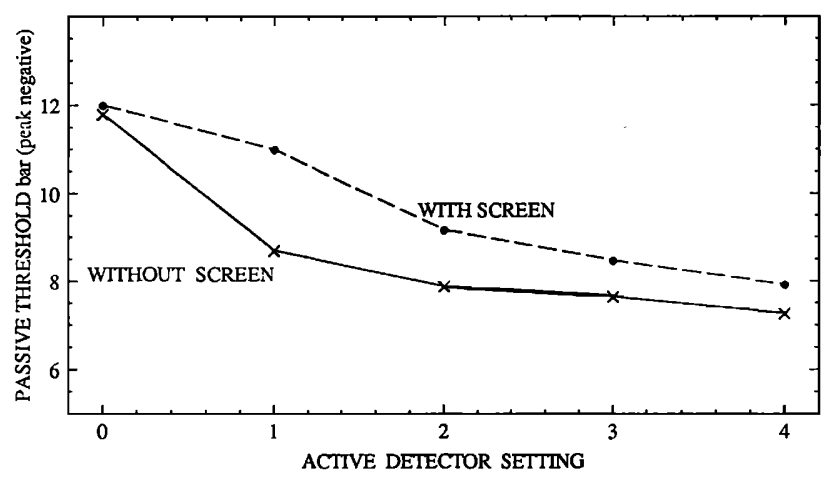

FIG. 9. Passive thresholds in the absence of streaming. A Saran Wrap ${ }^{\mathrm{TM}}$ (less than $10 \mu \mathrm{m}$ thickness) screen in front of the active detector discourages the buildup of streaming flow, while remaining acoustically transparent. The observed thresholds are lower for settings associated with stronger active detector field (higher streaming velocities). The settings $1,2,3$, and 4 in fact correspond to streaming velocities $0.27,0.48,1.86$, and $3.25 \mathrm{~cm} / \mathrm{s}$, respectively, with the screen removed. Cavitation transducer: $10-\mu \mathrm{s}$ pulse, $1-\mathrm{kHz}$ PRF, confocal setting. Test cell: $0.984-\mu \mathrm{m}$ polystyrene spheres, $1.4 \times 10^{7}$ particles/cc, water ( $80 \%$ dissolved air saturation). the number of particles added into the focal zone during the $10-\mu$ s interval is just 2 . This figure at the highest particle concentration would be about 128 particles. However, during the $10 \mu$ s on time the active detector could be affecting about 11000 particles present in the common focal region (cylinder of diameter $0.2 \mathrm{~mm}$ and length $2 \mathrm{~mm}$ ). It could, therefore be processing these particles to increase their nucleation potential.

A possible scenario: As observed earlier, the surface of the polystyrene latex particles is smooth down to $500 \AA$. Even so, they could have nano-scale gas pockets on the surface. This gas could be due to the imperfect wetting of the sphere, or the adsorbed nonpolar constituents desorbing when the particle enters the reduced pressure region of the main cavitation transducer. If the observed lower thresholds are to be possible, the nano-gas-dots must aggregate to form sufficiently larger gas patches. (For a nano-gas-dot of size 50 $\mathrm{nm}$, according to the model suggested below, the estimated threshold would be around 60 bar peak negative.) Is it possible for the active detector field to promote such cooperative aggregation?

In water, the wavelengths at 30 and $0.75 \mathrm{MHz}$ are $50 \mu \mathrm{m}$ and $2 \mathrm{~mm}$, respectively, while the largest particles we have used are less than $1 \mu \mathrm{m}$. So the long-wavelength assumption is valid and a particle essentially feels a uniform environment over its extent. Also, in the focal zone of a focused transducer one can assume that the waves are nearly plane. In the case of the active detector operating at a setting of $46 \mathrm{~V}_{\mathrm{pp}}$ and a pulse width of $10 \mu \mathrm{s}$, one would have about 300 full waves passing any point in the focal region. The estimated pressure level at this setting is about 0.5 bar peak negative (Ref. 3 ). Operating at $30 \mathrm{MHz}$ in water, this would give rise to a particle (fluid parcel) acceleration of about $6.47 \times 10^{6} \mathrm{~m} / \mathrm{s}^{2}$, or equivalently $6.5 \times 10^{5} \mathrm{~g}$ units. At any given point a particle heavier than water will inertially lag the acceleration, while a lighter particle will move in the direction of acceleration. In the present case we are considering the polystyrene spheres whose density is $1.05 \mathrm{~g} / \mathrm{cc}$, which are reasonably density matched with water. Air density, on the other hand, is about $1.2 \times 10^{-3} \mathrm{~g} / \mathrm{cc}$. This density contrast, a factor of 830 , will enhance the kinetic buoyancy and urge the nanogas-dots toward the leading stagnation region on the sphere where they could agglomerate and form a gas cap. One can visualize these gas caps as lenslike regions located at the extremities of an oscillating (to and fro) spherical particle. A cavitation event can then occur when the force due to surface tension on the perimeter of the gas cap is overcome by the tensile forces effective on the gas region. (The whole particle is in the tensile environment.) Thus separated air mass can give rise to cavitation and be detected.

In the case of the polystyrene spheres of mean diameter $0.984 \mu \mathrm{m}$, when the active detector is set to give a streaming velocity of $3.25 \mathrm{~cm} / \mathrm{s}$, the measured thresholds is 5 bar peak negative. The surface tension $(0.073 \mathrm{~N} / \mathrm{m})$ force acting on the perimeter of a circle of diameter $0.984 \mu \mathrm{m}$ can be overcome by a tensile force due to a pressure of just 3 bar peak negative acting on the corresponding projected area. Here we have assumed a gas patch of the same diameter as the particle. In reality, the gas patch would be smaller than the 
particle dimensions. This would invariably give rise to thresholds higher than the lower limit corresponding to a gas patch as large as the particle diameter.

\section{F. Thresholds and particle size}

How the particle size affects the measured thresholds is studied to see if the results corroborate the analysis. Figure 10 shows how the passive thresholds vary as a function of the streaming velocities for different particle sizes, while Fig. 11 gives the active detector results. We use three particle sizes, essentially monodispersed having mean diameters of 0.245 , 0.481 , and $0.984 \mu \mathrm{m}$. The particle number density is maintained the same at $1.9 \times 10^{8}$ particles/cc. The dissolved air saturation in the test cell is maintained around $70 \%$.

When the active detector is switched off, it does not participate in the cavitation process; the thresholds are solely due to the main cavitation field. The observed passive thresholds are around 17 bar peak negative with the larger particles giving a slightly smaller value (Fig. 10). It should be noted that if the main cavitation transducer were to act alone, in order to give rise to the acceleration levels as high as those due to the active detector (set to yield a streaming of $3.25 \mathrm{~cm} / \mathrm{s}$ ) operating at $30 \mathrm{MHz}$ and a pressure amplitude of $0.5 \mathrm{~atm}$ peak negative, the pressure amplitude of the main cavitation transducer ought to be higher by a factor of 30 so as to keep the "frequency-pressure" product the same; passive thresholds in the absence of the active detector field should be around 15 bar peak negative. Figure 10 corroborates this expectation convincingly.

The effect of particle size becomes more pronounced at the higher streaming velocities (stronger active detector fields). Also, smaller particles are associated with greater thresholds.

\section{G. Effect of gas concentration}

Figure 12 shows the threshold dependence on the dissolved air saturation. Clean water was degassed to the de-

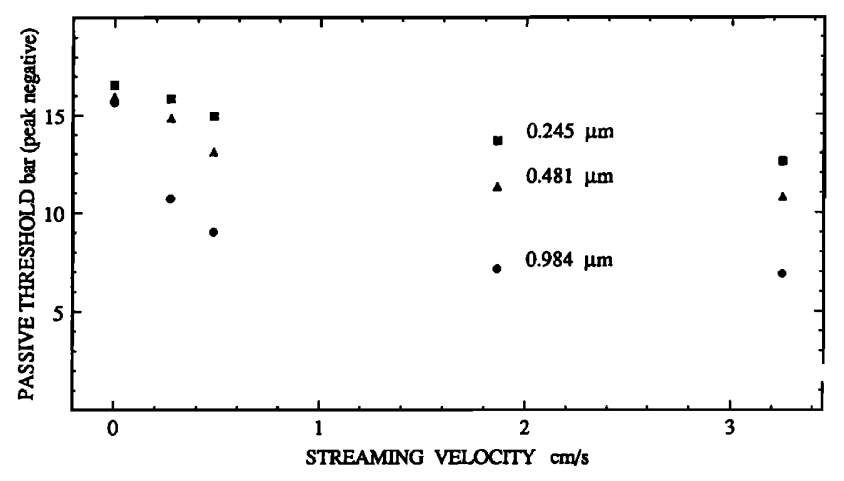

FIG. 10. Particle size effect on passive thresholds. The monodispersed polystyrene latex microspheres of three different sizes were used: $0.254,0.481$, and $0.984 \mu \mathrm{m}$. In the plot they are represented by solid squares, triangles, and circles, respectively. The particle number density was maintained the same $-1.9 \times 10^{8}$ particles/cc. Passive thresholds decrease with increase in particle size, and in all the cases thresholds invariably decrease with increase in streaming velocity (stronger active detector field). With the active detector switched off, the passive threshold for different particles has almost the same value. Cavitation transducer: 10- $\mu$ s pulse, $1-\mathrm{kHz}$ PRF, confocal setting. Test cell: water (70\% dissolved air saturation).

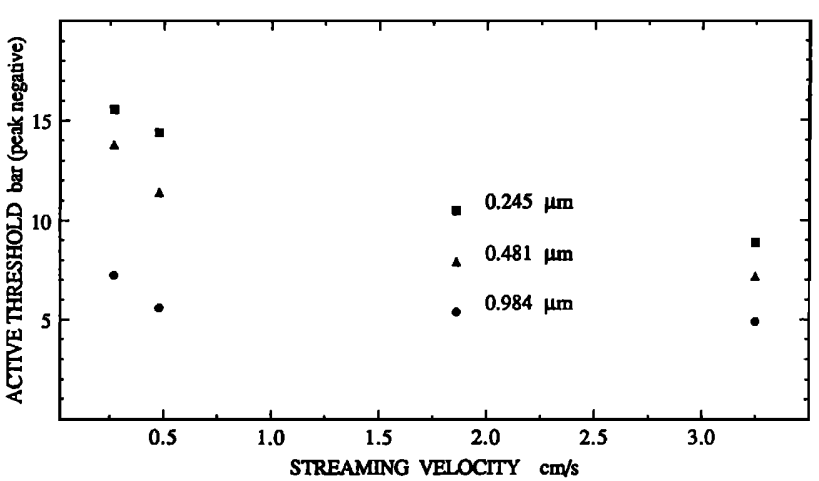

FIG. 11. Particle size effect on active thresholds. The active threshold variation as a function of the particle size and the streaming velocity. Identical test conditions as Fig. 10.

sired air saturation before filling up the test cell. We used polystyrene particles of mean diameter $0.984 \mu \mathrm{m}$ at a number density of $1.4 \times 10^{7}$ particles/cc. Both the active and the passive thresholds were measured as a function of the streaming velocity. Thresholds are lower while the active detector is on. However, the observed thresholds are higher when the dissolved air concentration is lower, suggesting the difficulty of formation of the gas cap of adequate size. The threshold variation with respect to an increase in streaming (stronger acoustic field) is less pronounced at lower gas saturations, further supporting the previous statement. Both the active and the passive trends were similar.

\section{H. Effect of the acoustic parameters}

The range of variation of the acoustic parameters is primarily limited by the capabilities of the apparatus used. These experiments were conducted using $0.245-\mu \mathrm{m}$ polystyrene spheres at a number density of $9.6 \times 10^{8}$ particles/cc. The dissolved air saturation in the test cell was around $75 \%$. Both the active and the passive thresholds were measured. The active detector setting in these experiments was kept fixed at $10-\mu$ s pulse width and $46 V_{p p}$. Only the main cavita-

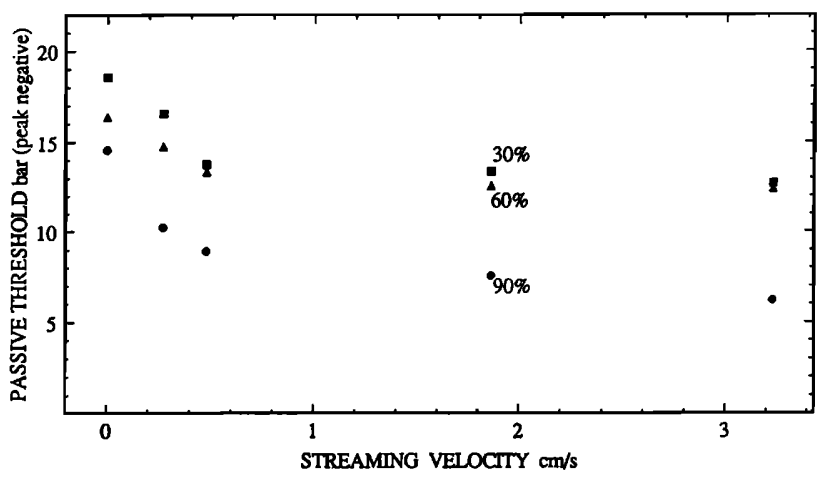

FIG. 12. Passive thresholds for various dissolved air concentrations. Thresholds decrease with increasing gas (dissolved air) concentration. The variation is less pronounced at lower dissolved air concentrations. Thresholds also decrease with increased streaming velocity. Streaming velocity is determined by the active detector setting as mentioned in the text. In the plot, the dissolved air concentrations of $30 \%, 60 \%$, and $90 \%$ are represented by solid squares, triangles, and circles, respectively. Cavitation transducer: $10-\mu$ s pulse, $1-\mathrm{kHz}$ PRF, confocal setting. Test cell: $0.984-\mu \mathrm{m}$ polystyrene spheres, $1.4 \times 10^{7}$ particles $/$ cc. 
tion transducer parameters were varied.

Figure 13 shows how the thresholds vary as the pulse width is increased. The cavitation transducer PRF was kept fixed at $1 \mathrm{kHz}$. Three pulse widths were tried: 10, 20, and 30 $\mu \mathrm{s}$. The plots indicate that initially the thresholds decrease as the pulse widths increase. However, for longer pulses the plots suggest that the thresholds may level off. It is to be remembered that the active detector pulse of $10 \mu$ s coincides with the first $10 \mu$ s of the cavitation pulse, leaving the remainder of the cavitation pulse exposed only to the residual effects of the active detector. Any cavitation occurring during this aftermath of the active pulse can only be picked up by the passive detector; the active detector is deaf during this time. Leveling is, therefore, more pronounced with active thresholds than for passive thresholds.

Figure 14 shows how the thresholds decrease as the PRF is increased. In these plots the pulse width is kept constant at $10 \mu \mathrm{s}$, and both the detectors indicate similar trends in thresholds. Increasing PRF implies greater exposure to the active detector activity.

\section{Pulse probing}

The cavitation pulse can be probed with the active detector pulse by varying the arrival time of the interrogating pulse at the focus. The active detector was arranged confocally with respect to the main cavitation transducer. The active detector setting was held at $5-\mu$ s pulse width, $1-\mathrm{kHz}$ $\mathrm{PRF}$, and $46 \mathrm{Vpp}$. The test cell dissolved air saturation was around $80 \%$, and the cavitation environment has $0.245-\mu \mathrm{m}$ polystyrene particles at a number density of $9.6 \times 10^{8}$ particles/cc. The main cavitation transducer is operated at $10-\mu \mathrm{s}$ pulse width and 1-kHz PRF.

Figure 15 shows the performance of the detectors. The thresholds are the lowest when the interrogating pulse arrives while the cavitation pulse is at the focus. The kinetic buoyancy effect should be the greatest at this time coincidence. Advancing the detector pulse by $5 \mu$ s also yields low thresholds, as the active detector presence is still not fully decoupled from the cavitation pulse. On the other hand,

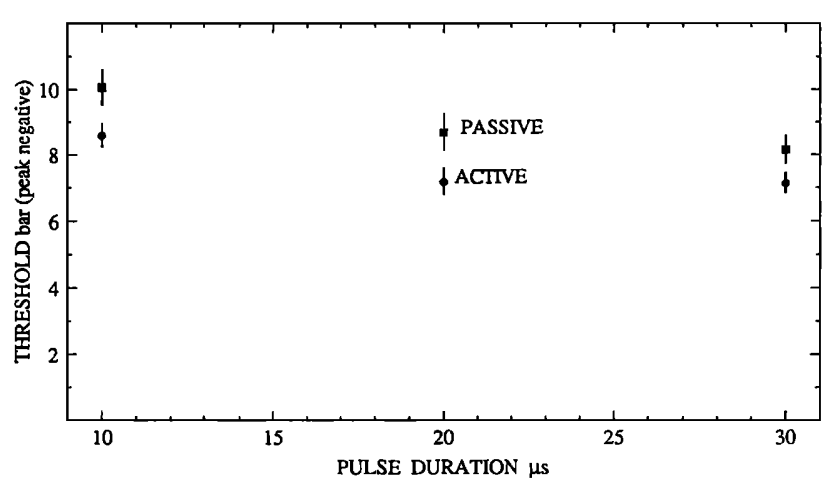

FIG. 13. Thresholds for constant PRF. For a given PRF, as the pulse width increases, thresholds decrease. This is due to the increased number of cycles available in the pulse to cause cavitation. The trend is borne out by both the detectors. Active detector setting: $10-\mu$ s pulse, $46 \mathrm{~V}_{\mathrm{pp}}, 1-\mathrm{kHz}$ PRF. Cavitation transducer: $1 \%, 2 \%, 3 \%$ duty cycles. Test cell: $0.245-\mu \mathrm{m}$ polystyrene spheres, $9.6 \times 10^{8}$ particles/cc, water ( $75 \%$ dissolved air saturation).

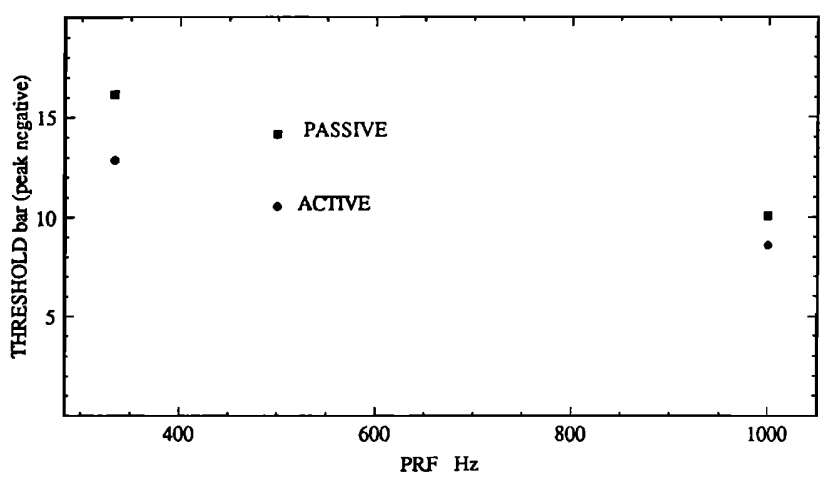

FIG. 14. Thresholds for constant pulse width. For a given pulse width, as the PRF increases, thresholds decrease. Both the detectors show similar variation in the measured thresholds. In the plot, passive thresholds are indicated by solid squares and active thresholds by solid circles. Active detector setting: $10-\mu$ s pulse, $46 \mathrm{~V}_{\mathrm{pp}}$, same $\mathrm{PRF}$ as the main transducer, confocal setting. Cavitation transducer, $10-\mu$ s pulse, and 333,500 and $1000-\mathrm{Hz}$ PRF's. Test cell: $0.245-\mu \mathrm{m}$ polystyrene spheres, $9.6 \times 10^{8} \mathrm{particles} / \mathrm{cc}$, water (75\% dissolved air saturation).

with increased detector delay, the active detector participation ceases quickly. Thresholds rise even at $10-\mu$ s delay, corresponding to the detector pulse just trailing the cavitation pulse. The usefulness of the active detector is quickly lost; it begins giving unreliably high thresholds and the error bars progressively increase. The passive detector, on the other hand, reads almost the same threshold levels as influenced by the residual streaming effects due to the active detector.

\section{J. Focal scan}

In this experiment the focal axis of the main cavitation transducer is probed. Figure 16 shows the results. The location of the active and the passive detectors is kept fixed, and the main cavitation transducer is retracted along its focal axis (positive distance in the plot). The active detector setting is kept at $10-\mu$ s pulse width, $1-\mathrm{kHz}$ PRF, and $46 \mathrm{~V}_{\mathrm{pp}}$. The test conditions are same as the previous setup.

Active thresholds are lowest at the confocal setting. At

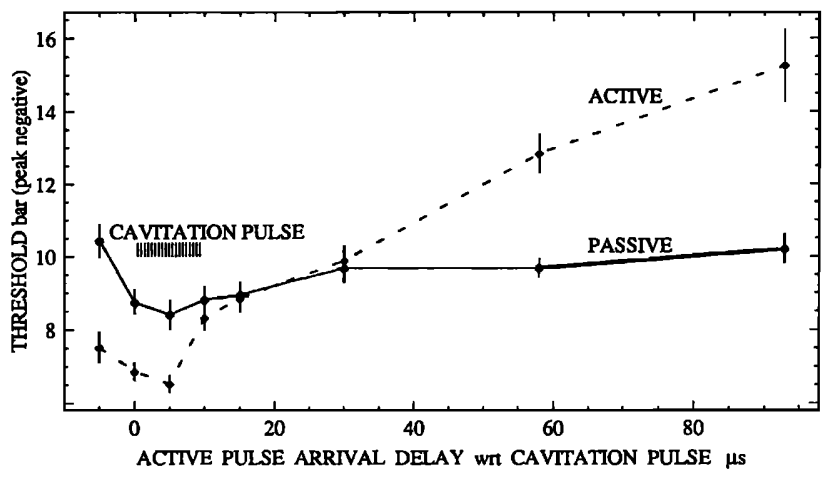

FIG. 15. Temporal scan. When the arrival of the active detector pulse at the common focus is delayed with respect to the main cavitation pulse, interaction effects are seen in the measured thresholds. The sensitivity characteristics of the detectors also stand in contrast. Active detector setting: 5- $\mu$ s pulse, $46 \mathrm{~V}_{\mathrm{pp}}, 1-\mathrm{kHz}$ PRF. Cavitation transducer: 10- $\mu$ s pulse, 1-kHz PRF. Test cell: $0.245-\mu \mathrm{m}$ polystyrene spheres, $9.6 \times 10^{8}$ particles $/ \mathrm{cc}$, water $(80 \%$ dissolved air saturation). 


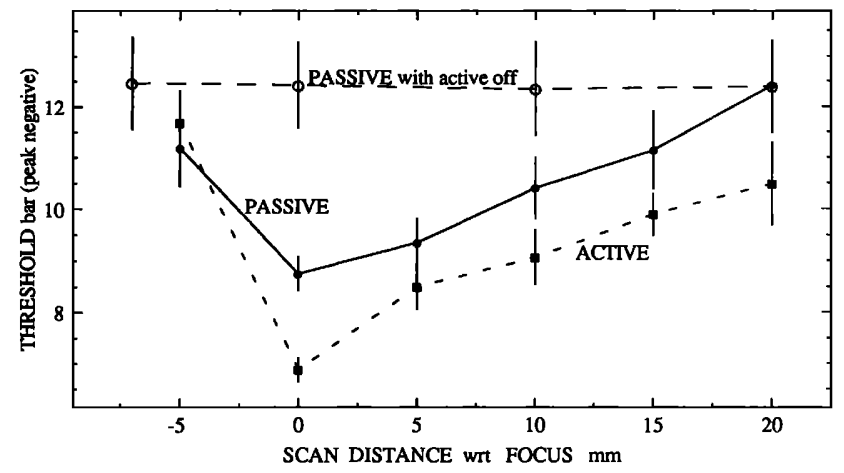

FIG. 16. Focal scan. When the active detector looks for events at different locations along the focal axis of the main cavitation transducer, measured thresholds vary as shown. Simultaneous detection by the passive transducer also indicates a similar variation in thresholds. However, the passive transducer does not indicate any variation in the measured thresholds (scanned along the main cavitation focal axis) when the active detector is switched off. Active detector setting: $46 \mathrm{~V}_{\mathrm{pp}}, 10-\mu$ s pulse, 1-kHz PRF. Cavitation transducer: $10-\mu$ s pulse, 1-kHz PRF with axial traverse. Test cell: $0.245-\mu \mathrm{m}$ polystyrene spheres, $9.6 \times 10^{8}$ particles/cc, water ( $80 \%$ dissolved air saturation).

the distance of $-5 \mathrm{~mm}$, the active detector focus is behind the main cavitation focus and in the converging cone of the latter. The thresholds are the highest. The kinetic buoyancy contribution to nucleation and the streaming effect are expected to be greatest only at the confocal setting. Thresholds increase gradually, primarily due to the gradual fall in cavitation peak pressure downstream. Contrary to the previous expectation, the passive detector follows the threshold changes quite reliably. It was thought that the passive detector, being a wide field of view device, could listen to the events if they occurred at the focus, and were missed by the active detector because of its nonconfocal setting. An instance of this is seen at the scan distance of $-5 \mathrm{~mm}$, where the passive threshold is lower than the active threshold. The plots demonstrate the active detector participation in the cavitation process.

To establish how well the passive detector performs, the active detector was switched off and the passive thresholds measured, while retracting the main cavitation transducer along the focal axis, as above. The passive thresholds were remarkably consistently. In fact, at the $20-\mathrm{mm}$ scan point, the active detector is completely out of the focal zone of the main cavitation transducer. Here, the passive thresholds coincide with the previous value. This would mean that at this location the active detector ceases to participate in the cavitation process. Once past the focus, all through the downstream scan the active and the passive trends are almost parallel.

\section{SUMMARY AND CONCLUSIONS}

\section{A. Comparison of active and passive detectors}

Both the active and the passive detectors have performed well in the course of the present research. They have followed the threshold trends equally well. The active detector is a focused device, and can be used to probe a narrow region of space for cavitation activity. It is rather insensitive to cavitation activity outside of its focal field of view. Therefore, the active detector would help in probing the space for locating the region of cavitation activity. However, for the active detector to detect cavitation, the interrogating pulse must be present while the cavitation is occurring. In this sense the passive detector, like the ear, remains a constant sentinel. Also, the passive detector has a wide field of view and can watch over a larger region of space to monitor cavitation. Being a passive listener, it relies on the strength of the sound source to speak to it. The active detector relies on the received echo from the region of interrogation and is, in fact, found to affect the cavitation process. It is, therefore, a mildly invasive technique of measurement, while the passive detector is truly passive.

As seen on the scope screen, the backscattered signal that constitutes the background for the active detector is fairly constant in strength but is filled with activity and the occasionally transiting elephants (wide, slow-moving echoes). Once the operator gets to this bustling background, event recognition is quite easy. In the case of the passive detector the reverberant sound constitutes the background, which could be significantly modified by the test chamber design: This background level rises continuously as the cavitation signal is ramped up. However, within this background there is no activity, the region is a steady glow, and should cavitation events smaller than the background occur, they would be missed. It is easier to get used to the passive detector, and the eye strain is less for prolonged periods of observation. Even so, if there is a wide variation in the character of the manifested events, the operator's visual reference is constantly changing, and precision may suffer. On the other hand, if the background scatter in the case of the active detector becomes excessively high, the detector would be rendered nearly useless.

\section{B. Active detector and cavitation}

We have found that the active detector does affect the cavitation process. The mechanics of participation can be understood in terms of the two modes of action given below.

(i) The active detector can convect in particles into the cavitation zone. The number of particles convected in depends on the number density of the particles in the test cell, the pulse-width voltage setting of the active detector (which determines the streaming velocity), and the time duration during which the particle flux is effective. The last factor is determined by the on-time of the cavitation transducer and time/space setting of the active detector relative to the cavitation transducer.

(ii) The active detector acoustic field helps cultivate nuclei on the particles. High acoustic frequencies cause high particle (fluid parcel) accelerations even at moderate- or low-pressure amplitudes. These high accelerations combined with the density contrast gives rise to the kinetic buoyancy forces that promote the formation of gas patches through the cooperative aggregation of the nano-gas-dots presumed present on the surface of the smooth spherical particles. The nano-gas-dots could be due to the imperfect wetting of the particles, or the adsorbed nonpolar constituents 
desorbing when the particle encounters reduced pressures in the cavitation field. More work needs to be done in this area, especially using particles other than the polystyrene latex microparticles.

Rectified diffusion as a possible mechanism for bubble growth under the influence of the active detector appears to be unlikely based on the simulations run by Dr. Charles Church. $^{5}$

There is one possible mode of participation that needs further study. It is possible that a strong cross streaming flow may hinder the flushing off of potential nuclei along the focal axis of the main cavitation transducer, thus allowing them a greater time exposure to the cavitation field ${ }^{6,7}$ and so facilitate cavitation at lower pressures. It takes but one fruitful nucleus to cause a cavitation event.

\section{ACKNOWLEDGMENTS}

We are grateful to Professor B. T. Chu for helpful discussions in clarifying and consolidating the thoughts about cavitation and calibration. Dr. Charles Church of NCPA was gracious enough to check and rule out the possibility of gas accretion through rectified diffusion under the influence of the active detector. We especially acknowledge his help. This research was funded through NIH grant 5R01-32794.

\section{APPENDIX A: CALIBRATION OF THE MAIN CAVITATION TRANSDUCER}

In calibrating the transducer, its voltage to acoustic pressure conversion ratio is sought, which enables us to estimate the peak negative pressure at the focus once the input voltage amplitude to the transducer is known. Very briefly, the procedure consists in determining two ratios-the total acoustic power produced at the focus to the voltage input to the transducer (using the force balance), and the ratio of the peak intensity to the total acoustic power at the focus (as determined from the DAPCO ${ }^{\mathrm{TM}}$ measurements to be mentioned). A cross multiplication of these two ratios leads us directly to the desired pressure conversion factor (Ref. 3).

The radiation force balance needs a well-characterized target, and a "perfectly" absorbing or "perfectly" reflecting target simplifies the calculations. We use a "perfectly" reflecting target simplifies the calculations. We use a "perfectly" reflecting target. In a $1.5-\times 2-\times 1 / 4$-in.-thick Plexiglas piece, $1-\times 1.5-\times 1 / 8$-in.-deep cavity is milled out on the reflecting face. This cavity is sealed with a very thin stainless steel sheet ( $12 \mu \mathrm{m}$ thick), which is acoustically transparent for our purpose. Suitable ballast weights, in the form of stainless steel shim strips, are bonded to the sides, to render the target nearly neutrally buoyant. When immersed in water this air pocket serves as a perfectly reflecting interface. This perfectly reflecting window is arranged to suspend at an angle of $45^{\circ}$ to the incoming horizontal acoustic beam. Even under deflection by radiation force, the $45^{\circ}$ window orientation with respect to the acoustic beam is maintained using the bifilar suspension. Four nylon monofilaments are used to secure the suspension, which also provides additional torsional stability through the use of a wider roof for suspension. The projected area of the target to the incoming beam is 1 in. $\times 1$ in. (wavelength at $0.75 \mathrm{MHz}$ is $2 \mathrm{~mm}$ ). In our case the problem of standing wave formation is obviated because we test only in the pulse mode. Should a continuous wave testing be required, one needs to provide an absorbing surface (rho-c ${ }^{\mathrm{TM}}$ platform) at the bottom of the target to suppress the reflected radiation. With the continuous mode there is an additional problem of severe streaming. In order to minimize the streaming effects one needs to cage suitably the target and partition the flow. In spite of these precautions, the continuous mode of testing yields a linear plot (total intercepted acoustic power versus the drive voltage squared) only for low-drive voltages, and therefore the calibration remains valid only for low-drive voltages.

To commence calibration in the pulse mode, the transducer is set to the drive parameters mentioned earlier, and the force balance is aligned to intercept the full beam at the focus of the main transducer. The deflection of the force balance is measured using a traveling telescope whose cross hairs are trained on the reference mark etched on the side of the target. Force balance deflection is noted for the full range of variation of driving voltage -10 to $300 \mathrm{~V}_{\mathrm{pp}}$ - to the transducer. Both rising and falling deflections were noted and no hysteresis was observed. The plot of the total acoustic power obtained from the deflection measurements versus the square of the input voltage is very linear, and its slope gives us the first required ratio (total acoustic power to the square of the input voltage, $W / \mathrm{V}_{\mathrm{pp}}{ }^{2}$ ).

The target in the displaced position is in equilibrium under the action of three forces: radiation force $F$, acting horizontally in the direction of the beam; tension $T$, along the length of suspension; and the weight of the target $\mathrm{mg}$, acting vertically downward. The radiation force can be expressed in terms of the target weight and the deflection geometry:

$$
F=m g d / \sqrt{\left[l^{2}-d^{2}\right]} \approx m g d / l \text { for } l \gg d,
$$

where $g$ is acceleration due to gravity, $980 \mathrm{~cm} / \mathrm{s}^{2} ; d$ is deflection in $\mathrm{cm}$; $l$ is suspension length, $10.6 \mathrm{~cm}$; and $m$ is weight in water, $0.95 \mathrm{~g}$.

The total acoustic power $W$ is given by

$$
W=F c=1.313 d \text { watts, }
$$

where $c$ is speed of sound in water, $1495 \mathrm{~m} / \mathrm{s}$.

Before the second ratio is determined, some more measurements are made using the DAPCO ${ }^{\mathrm{TM}}$ needle hydrophone. The test cell is filled with clean water from the main reservoir, and the $\mathrm{DAPCO}^{\mathrm{TM}}$ hydrophone is arranged along the focal axis of the main transducer with its sensing tip at the focus. The main transducer is driven with a voltage amplitude of $30 \mathrm{~V}_{\mathrm{pp}}$ and a $1 \%$ duty cycle. The transverse beam profile is obtained down to the first side lobe (which is quite weak compared to the main lobe); the following integration is performed, establishing the second ratio (peak intensity to total acoustic power, $I_{\text {peak }} / W$ ):

$$
\begin{aligned}
& I_{\text {peak }}=k v_{\mathrm{DAPCO}}^{2}, \\
& W=\int_{0}^{r} 2 \pi r I(r) d r=2 \pi k \int_{0}^{r} r v_{\mathrm{DAPCO}}^{2} d r,
\end{aligned}
$$

where $k$ is the constant of proportionality; $r$ is the radial distance from the focal axis of the cavitation transducer; and 
$v_{\text {DAPCo }}$ is the voltage measured by the DAPCO ${ }^{\text {mM }}$ needle hydrophone.

The pressure conversion ratio is established from a cross multiplication of the above-mentioned ratios, after substituting for the peak intensity in terms of the peak pressure, and the characteristic impedance of the medium.

While the DAPCO ${ }^{\mathrm{MM}}$ is still in the test cell, we measure the peak amplitudes of the pulse received by the DAPCO ${ }^{\mathrm{TM}}$, for the entire range of the voltage input to the main transducer. It is noticed that the main transducer pulse becomes moderately shocked for voltage inputs greater than $40 \mathrm{~V}_{\mathrm{pp}}$, so both the positive and the negative peaks of received pulse need to be measured. In this clean water environment, no cavitation was observed even at the highest input voltages to the main cavitation transducer.

It is possible to assume that the above pressure conversion factor gives us the positive peak for a given voltage input to the transducer, because the mildly shocked sine wave is not symmetric; it has a greater area above the time axis than below, and can be thought of as a superposition of a shocked sine wave (with all its harmonics) and a positive dc pressure shift. In this case the negative pressure peak can be estimated from the positive pressure peak given by the pressure conversion faetor using direct proportion (ratio of the positive peak to the negative peak of the received DAPCO ${ }^{\mathrm{TM}}$ pulse). A look-up table is prepared that gives the negative pressure peak corresponding to the input voltage to the transducer. As previously mentioned, the negative pressure amplitude is relevant to the cavitation threshold. A substitution calibration of the DAPCO ${ }^{\mathrm{TM}}$ needle hydrophone with respect to the reference PVDF membrane hydrophone using a common $750-\mathrm{kHz}$ focused transducer as a transmitter gave pressure estimates within one bar of those predicated by the force balance (with the above correction).

Over the entire testing period the calibration was checked twice, and no significant change was measured. To monitor the working condition of the transducer, its capacitance was measured every so often and was found to be constant. The transducer was never left exposed to a water environment except for the duration of the test run (typically not more than $8 \mathrm{~h}$ ). Before conducting experiments involving duty cycles other than $1 \%$, the transducer calibration was repeated for that specific duty cycle. A change in duty cycle also required a recalibration of the data acquisition system.

\section{APPENDIX B: ESTIMATION OF STREAMING VELOCITIES}

Streaming velocities can be experimentally measured using a rather straightforward adaptation of the time-of- flight technique. The submicronic particles are added to the test cell, which is filled with clean water, and the active detector is switched on to establish its streaming field. On the scope trace one occasionally observes a strong echo of the same time width as the interrogating pulse, rising tall above the background and slowly ambling its way down the time axis. As previously mentioned, such an echo is called an "elephant." The elephant is possibly due to a rather successful cooperative aggregation of the nano-gas-dots on a carrier particle. It is not the backscattering from the particle itself, because there are more than a million particles in the path of the pulse and one would have a screen full of elephants all the time. These elephants are not observed in the clean water. However, if the circulation pump is switched on, one occasionally observed rapidly transiting bubbles (resembles wildly running enormous elephants).

Since we use polystyrene particles whose density $(1.05$ $\mathrm{g} / \mathrm{cc}$ ) does not differ very much from the host (water), and the particle size is also very small (less than $1 \mu \mathrm{m}$ ), they are fully entrained in the flow and, therefore, the elephant speed closely approximates the average streaming velocity. The speed of the elephants seems to be correlated with the active detector voltage. Their speed increases with the increase in voltage and stays constant for a given voltage and pulse width. One can therefore measure the average streaming velocity as a function of the detector voltage and the pulse width.

The speed of the elephant is determined by measuring the distance traversed from the time-of-flight difference between the echo separation and the time interval between the triggers causing the echoes (Ref. 3 ).

${ }^{1}$ R. A. Roy, S. I. Madanshetty, and R. E. Apfel, “An acoustic backscattering technique for the detection of transient cavitation produced by microsecond pulses of ultrasound," J. Acoust. Soc. Am. 87, 2451-2458 (1990). ${ }^{2}$ A. A Atchley, L. A. Frizzel, R. E. Apfel, C. K. Holland, S. I. Madanshetty, and R. A. Roy, "Thresholds for cavitation produced in water by pulsed ultrasound," Ultrasonics 26, 280-285 (1988).

${ }^{3}$ S. I. Madanshetty, "Microcavitation studies using active and passive acoustic detection," doctoral dissertation, Yale University (1989).

${ }^{4}$ E. A. Neppiras, “Acoustic cavitation,” Phys. Rep. 61, 210 (1980).

${ }^{5} \mathrm{C}$. C. Church, "A method to account for acoustic microstreaming when predicting bubble growth rates produced by rectified diffusion," J. Acoust. Soc. Am. 84, 1758-1764 (1988).

${ }^{6} \mathrm{G}$. W. Willard "Focusing ultrasonic radiators," J. Acoust. Soc. Am. 21, 360-375 (1949).

'G. W. Willard, "Ultrasonic induced cavitation in water: A step-by-step process," J. Acoust. Soc. Am. 25, 669-686 (1953). 